\title{
Corela
}

Cognition, représentation, langage

HS-20 | 2016

L'Implicite

\section{Sans les mots : pédagogie de la prosodie sémantique en classe de FLE}

Chantal Rittaud-Hutinet

\section{OpenEdition}

1 Journals

Édition électronique

URL : https://journals.openedition.org/corela/4706

DOI : $10.4000 /$ corela.4706

ISSN : 1638-573X

Éditeur

Cercle linguistique du Centre et de I'Ouest - CerLICO

Référence électronique

Chantal Rittaud-Hutinet, «Sans les mots : pédagogie de la prosodie sémantique en classe de FLE », Corela [En ligne], HS-20 | 2016, mis en ligne le 15 décembre 2016, consulté le 20 juin 2021. URL:

http://journals.openedition.org/corela/4706 ; DOI : https://doi.org/10.4000/corela.4706

Ce document a été généré automatiquement le 20 juin 2021.

\section{(c) (i) (2) (2)}

Corela - cognition, représentation, langage est mis à disposition selon les termes de la licence Creative Commons Attribution - Pas d'Utilisation Commerciale - Partage dans les Mêmes Conditions 4.0 International. 


\title{
Sans les mots : pédagogie de la prosodie sémantique en classe de FLE
}

\author{
Chantal Rittaud-Hutinet
}

"Most of the time our utterances are automatically interpreted as speech acts : as assertions, conjectures and testimonies ; as orders, requests and pleas ; as threats, offers and promises. Surprisingly, the cognitive correlates of

this essential component of human communication have received little attention".

Kissine M., 2013

\section{Introduction}

1 Pour les linguistes, il est clair que l'énonciation spontanée n'est pratiquement jamais neutre, strictement informative et objective, mais infusée de notre subjectivité, de nos doutes, goûts, humeurs, caractères, sentiments, etc. (cf. Gabrys-Barker D. \& al. (éds) 2013, Plantin Ch. \& al. (éds) 2000). A l'oral, nombreuses sont les stratégies discursives non lexicales de l'énonciateur, et particulièrement celle de l'implicite de l'énonciation, domaine de la phonopragmatique. En effet les signes vocaux autorisent des actes de langage (cf. Barth-Weingarten D. \& al. (éds.) 2010a et 201b, Brichet C. \& al. 2003, Carston R. 2009, Hirschberg J. 2002, Marti L. 2006, Rittaud-Hutinet C. 2006, Wennerstrom A. 2001) qui remplacent, secondent ou doublent les mots, et donc permettent de modaliser l'énoncé sur lequel ils apparaissent, de le valider ou de le biaiser, de l'accentuer ou de l'affaiblir, de l'annuler ou même de le contredire; ce que les écrivains s'efforcent, à l'écrit, de restituer, comme dans l'exemple (1) :

2 (1) "- J'ai bien peur que le poulet soit trop cuit... dit-elle en servant Maigret. 
3 Et le ton était le même que pour dire, par exemple : «J'ai peur de tout ! Je ne sais pas ce qui se passe! Sainte Vierge, protégez-moi !»²

4 Aussi, étant donné son importance sur la qualité de l'intercompréhension et de l'intercommunication, la couche vocale gagnerait à trouver des mises en application plus larges dans les face-à-face pédagogiques, de même que dans les méthodes et manuels d'enseignement du français comme langue étrangère. Malheureusement, trop d'ouvrages se contentent encore à cet égard d'une profession de foi affichée dans l'introduction mais guère suivie de propositions effectives dans les unités pédagogiques. De plus, il arrive que les règles et fonctionnements de cette composante $\mathrm{du}$ discours en interaction soient absents de la formation des enseignants. Lorsque c'est le cas, la méconnaissance de la constitution acoustique, des sens des signes vocaux et de leurs emplois, comme la pauvreté des outils mis à disposition par les manuels, amènent souvent les enseignants à en rester en classe de langue au vœu pieux, non seulement parce qu'ils ont l'impression d'avoir affaire à des phénomènes changeants (cf. Portes $\mathrm{C}$. \& Bertrand R. 2011), mais aussi parce qu'ils trouvent ces unités - bien que codées difficiles à aborder en raison de leur fugacité.

Pour tenter de remédier à cette situation, après avoir résumé ce que sont les signes vocaux, leur importance sera montrée au travers de trois séries d'exemples significatifs: des "oh" et des "ah" dits par un locuteur (qui n'est ni comédien ni linguiste), "vous c'est vous" joué par des acteurs dans deux films, et deux signes vocaux à objectif méta-discursif, présents dans un dialogue non préparé. Puis seront rappelées rapidement les règles principales à appliquer pour ce type de mise en œuvre dans la classe de français comme langue étrangère.

\section{Les signes vocaux : qu'est-ce que c'est? Qu'en fait- on?}

6 Si l'on peut répondre à ce que n'a pas dit l'interlocuteur, c'est parce que des signes vocaux lui ont servi à faire comprendre la visée réelle de son propos. Par leur discrétion, les signes vocaux offrent beaucoup de solutions en interlocution. Ils sont très fréquemment présents, qu'il s'agisse d'effets calculés lors d'oral préparé - voulus par l'orateur, appris par le comédien-, ou des intentions du locuteur en conversation libre, non surveillée, et ce même si l'effet produit n'est pas toujours égal au résultat attendu. Aussi, que ce soit pour se distancier de son propre énoncé ou au contraire l'affirmer avec force, attirer l'attention du destinataire, le persuader, le surprendre, relancer son intérêt, lui prendre ou lui donner la parole, anticiper ses réactions, lui faire croire quelque chose, l'enjôler, le mettre en difficulté, le faire rire, lui faire un reproche, lui donner la parole, éviter de le choquer, réfuter ses dire, modifier le rapport de places avec lui ou opérer une régulation interpersonnelle - et la liste n'est pas close !-, les Français ont énormément recours à l'implicite de l'énonciation pour faire comprendre ce qui serait soit trop long à décrire verbalement, soit difficilement exprimable avec le vocabulaire. Partant, les signes vocaux convertissent un schéma d'énoncé en message (cf. Avanzi M. \& al. 2011, Beyssade C. \& al. 2004, Hirschberg J. 2002) et orientent le travail de décodage (cf. Lacheret-Dujour A. \& al. 1999, Morel M.-A. \& al. 1998, Rittaud-Hutinet C. 2012, Stewart D. 2009). De plus, ces unités linguistiques vocales ne sont qu'exceptionnellement trans-langagières, même si évidemment les signifiés se retrouvent dans les autres langues. 
7 Il va de soi que leur présence est subordonnée au 'vouloir dire' du sujet parlant (cf. Rittaud-Hutinet C. 2008, 2011b ), qui peut préférer la sous-spécification à la clarté. Tout dépend donc des buts qu'il assigne à sa parole, de ce qu'il veut faire comprendre et/ou obtenir de l'interlocuteur, et aussi du fait qu'il peut puiser dans les ressources d'une seule ou au contraire de plusieurs des composantes de la langue et également avoir recours à des indices posturo-mimo-gestuels.

\section{Des exemples significatifs}

\subsection{Des "oh" et des "ah"}

\subsubsection{Le corpus : traits acoustiques et interprétations}

8 Sans autre précision, j'ai demandé à une personne de 'jouer' à sa guise avec les lexies "oh" et "ah", en les réalisant comme des énoncés complets, et de toutes les manières qui lui plaisaient. L'objectif était de vérifier jusqu'à quel point la déplétion lexicale, la faiblesse du sens sémantique est en mesure d'être compensée par la composante vocale (cf. Rossi M. \& al. 1981). L'informateur - B, 38 ans - a fourni une vingtaine d'occurrences, enregistrées en audio. D'autres productions sont encore possibles, comme les 3 "oh" entendus récemment dans un vieux film, Le fauteuil $47^{3}$, (cf. extrait audio F47 joint) qui, eux, sont joués par une actrice (cf. Godement-Berline R. 2014) dans une situation bien précise.

9 Etant donné que les dictions de B ont été réalisées hors situation (cf. par comparaison Camp E. 2006, Rittaud-Hutinet C. 2011c), comme il le dit lui-même c'est le jugement/le ressenti/le but etc. auquel il pensait chaque fois qui lui a permis de les dire de façon crédible, c'est-à-dire de telle sorte que les signes vocaux soient actualisés de façon 'juste'.

10 J'ai sélectionné 5 de ces "oh" et 5 de ces "ah" (cf. extraits audio : $2 a$ ab 2c 2d 2e $4 a 4 b 4 c$ $4 \mathrm{~d} 4 \mathrm{e}$ joints) pour faire passer un test interprétatif (enregistré en audio) à 4 personnes : 2 adultes (мо, Ma), un garçon de 13 ans (P), et le diseur (B) (pour ce dernier, 6 mois après son enregistrement des interjections). Consigne: décrire ce qu'ils comprennent en écoutant chacun des items. J'avais rédigé ma propre traduction (CRH) avant le passage du test, pour ne pas influencer les réponses des personnes testées, ni être influencée par ces dernières.

11 Les exemples sous (2) et (4) précisent les qualités acoustiques particulières à chaque diction ${ }^{4}$, relevées par analyse perceptuelle (voir les conventions d'écriture en fin d'article). Y est restitué l'essentiel des gloses, sous la forme suivante : en italiques, ce qui semble être les mots-clés de chaque interprétation; soulignées doubles, les informations explicites sur la recréation mentale de contexte; en petites majuscules, les références sensorielles ou affectives (vision, audition, sensation, ressenti) ; soulignés simples, les commentaires sur la réalisation sonore de l'informateur (B); souligné en vagues, les commentaires qui peuvent être attribuées à la diction ou au sens.

On remarquera que plusieurs types d'informations apparaissent, et qu'ils peuvent se combiner. Les tableaux (3) et (5) donnent une vision synoptique des traits constitutifs des signes vocaux. Pour ceux-ci il faut insister sur le fait que la complexité qu'ils font ressortir masque la réalité de l'oral, lequel prouve en situation réelle une compétence parfaite de l'énonciateur dans sa langue maternelle (et $a$ fortiori celle des enseignants !). 
signifiant du signe vocal : voyelle ultra-longue avec voix tremblée en seconde partie, Fo modulé montant-descendant $\mathrm{M}>\mathrm{H}>\mathrm{MH}$

interprétations données :

CRH: Il fait semblant d'avoir PEUR mais avec PLAISIR, comme quand on est dans le "Labyrinthe de la mort" à la fête foraine.

B : Oioioille y a beaucoup hein, c'est riche. Qu'est-ce que j'ai voulu dire là. /.../ une ANGOISSE un peu imagée un peu forcée un peu rigolote quoi pas très sérieuse, donc y a un mélange d'INQUIÉTUDE et d'humour ; plutôt dans le camp de l'humour.

Mo : $\mathrm{Y}$ a un peu de peur, comme quand on pénètre dans une antre dans une grotte, en même temps /... / on ÉCOUTE l'écho et on a quelque FRISSON on prend la dimension de la PEUR que l'on doit ou ne pas avoir.

14 (2b) oh [“? כ h]

signifiant du signe vocal : occlusive glottale initiale, voyelle ultra-longue seulement partiellement voisée au début et avec dévoisement progressif, fricative glottale non voisée finale, Fo modulé descendant $\mathrm{H}>\mathrm{MH}, \mathrm{dB}^{++}$

interprétations données :

$\mathrm{B}:$ /.../ étonnement qui est d'ordre physique

CRH : "C'est pas croyable, quelle découverte extraordinaire !" "Ça alors, c'est fou !"

Ma : Il en fait un peu trop là il exagère un peu; c'est un oh forcé, comme s'il voulait exagérer sa réaction, comme si c'est pas sincère. Le fait d'être épaté peut-être, mais comme si c'était pas naturel.

$\mathrm{P}$ : Surprise. /.../ on vient de voir un truc qu'on s'attendait pas. Plutôt une bonne surprise.

15 (2c) oh [oo]

signifiant du signe vocal : voyelle double, la première étant seulement partiellement voisée, Fo modulé descendant $\mathrm{H}>\mathrm{MB}$

interprétations données :

CRH : "Tiens, ça me surprend !" qu'on peut aussi traduire par : "Ah bon, vraiment ?"

B : "Tiens donc !" /.../ Un peu maniéré. /.../

Ma: "C'est un «oh» bien délicat. Il a l'air de ne pas y toucher. Y pourrait y avoir de l'étonnement mais là il prend des gants plutôt; pour je sais pas pour pas froisser, pour pas...

$\mathrm{P}:$ Un peu gracieux. /.../

(2d) oh [o::]

signifiant du signe vocal : voyelle longue, Fo modulé montant-descendant $\mathrm{MB}>\mathrm{H}>\mathrm{B}$

interprétations données :

$\mathrm{CRH}$ : Etonnement important vis-à-vis de ce dont il est question, avec une petite réserve sur ce qu'il en pense.

B : /.../ ADMIRATION feinte, suffisamment visiblement feinte pour pas faire illusion /.../

18 (2e) oh [“?o.]

signifiant du signe vocal : occlusive glottale initiale, voyelle semi-longue, Fo modulé descendant $\mathrm{H}>\mathrm{B}, \mathrm{dB}^{+}$

interprétations données :

CRH : "C'est un scandale!! Non, ça je le refuse catégoriquement ! !" S'il parle à un enfant : "C'est très mal, ce que tu as fait! ! Je suis très FÂCHÉ !"

B : /.../ C'est pas vraiment méchant mais c'est pour simuler la COLÈRE /.../ : "Oh qu'est-ce 
que c'est que cette histoire !", ce genre de choses. C'est un oh pour ma fille $((3$ ans $1 / 2))$ ça typiquement.

Ma : Là c'est de la stupeur, il est très choqué, plus que choqué

Mo : Alors là y a du scandale là on est scandalisé : "C'est pas possible! Ah pas toi !" enfin... y a de l'exaspération : "Ça fait trois fois que je te le dis! c'est pas possible! ? ah pas toi !". $\mathrm{P}:$ /.../ ÉNERVEMENT lors d'un jeu /.../ pas très sérieux (( = pas sérieusement énervé))

(3) tableau récapitulatif des traits pour les "oh" du corpus

\begin{tabular}{|c|c|c|c|c|c|c|c|c|}
\hline & $\begin{array}{l}\text { quantité } \\
\text { vocalique }\end{array}$ & $\begin{array}{l}\text { duplication } \\
\text { segmentale }\end{array}$ & $\begin{array}{l}\text { timbre et } \\
\text { tension } \\
\text { articulatoire }\end{array}$ & voisement & $\begin{array}{l}\text { consonne } \\
\text { non } \\
\text { phonol. }\end{array}$ & $\begin{array}{l}\text { hauteurs } \\
\text { tonales = } \\
\text { Fo } \\
\text { (par } \\
\text { rapport à } \\
\text { la tessiture } \\
\text { du } \\
\text { locuteur) }\end{array}$ & $\begin{array}{l}\text { intensité } \\
\text { (par } \\
\text { rapport } \\
\text { à la base } \\
\text { du } \\
\text { locuteur) }\end{array}$ & $\begin{array}{l}\text { stabilité } \\
\text { vibratoire }\end{array}$ \\
\hline (2a) & [ ] & & & & & $\begin{array}{l}\text { montant- } \\
\text { descendant } \\
\mathrm{M}>\mathrm{H}>\mathrm{MH}\end{array}$ & & $\begin{array}{l}\text { voix } \\
\text { tremblée } \\
(2 \mathrm{e} \\
\text { partie })\end{array}$ \\
\hline (2b) & {[]} & & [0] & $\begin{array}{l}\text { voisement } \\
\text { initial } \pm \text { puis } \\
\text { dévoisement } \\
\text { progressif }\end{array}$ & $\begin{array}{l}\text { [?] initial } \\
{[\mathrm{h}] \text { final }}\end{array}$ & $\begin{array}{l}\text { descendant } \\
\mathrm{H}>\mathrm{MH}\end{array}$ & $\mathrm{dB}^{++}$ & \\
\hline (2c) & & [oo] & & $\begin{array}{l}\text { voisement } \\
\text { initial } \pm\end{array}$ & & $\begin{array}{l}\text { descendant } \\
\mathrm{H}>\mathrm{MB}\end{array}$ & & \\
\hline (2d) & {$[::]$} & & & & & $\begin{array}{l}\text { montant- } \\
\text { descendant } \\
\mathrm{MB}>\mathrm{H}>\mathrm{B}\end{array}$ & & \\
\hline (2e) & [.] & & & & [?] initial & $\begin{array}{l}\text { descendant } \\
\mathrm{H}>\mathrm{B}\end{array}$ & $\mathrm{dB}^{+}$ & \\
\hline
\end{tabular}

19 (4a) ah [? $\left.\mathrm{a}^{\tilde{\varepsilon}} \mathrm{h}\right]$

signifiant du signe vocal: occlusive glottale initiale, voyelle ultra-longue avec écartement forcé des commissures - ce qui correspond à une partie du geste articulatoire de la labialité de type vocalique (à noter : cela donne la sensation que B sourit) - et nasalisation partielle, fricative glottale non voisée finale, Fo modulé montant-descendant $\mathrm{IH}>\mathrm{H}^{+}>\mathrm{MB}, \mathrm{dB}^{+}$

interprétations données :

CRH : « Ça, ça fait PLAISIR/DU BIEN à ENTENDRE/VOIR/constater ! je suis SATISFAIT d'ENTENDRE/ de voIR/de constater ça!»

B : c'est un peu ironique mais c'est peut-être parce que c'est ma propre voix j'ai un peu de mal. Un étonnement un peu feint, un peu forcé. 
Ma : Contentement, SATISFACTION. Quelqu'un d'heureux qui se réjouit.

Mo: /.../ une surprise qui indique une certaine satisfaction /.../ on apprécie comme quand on voIT un beau tableau quand on ENTEND une remarque positive et intéressante $\mathrm{P}: / . . . /$ enfin on vient de comprendre après vraiment pas avoir compris du tout.

signifiant du signe vocal: occlusive glottale initiale, voyelle ultra-longue seulement partiellement voisée au début et avec dévoisement progressif, fricative glottale non voisée finale, Fo modulé descendant $\mathrm{H}>\mathrm{MH}, \mathrm{dB}^{++}$

interprétations données :

CRH : "C'est pas croyable, quelle découverte, c'est ChOuETTE/EXTRAORDinaiRe!" ou, dit autrement : "Ça alors, c'est fou/fabuleux!"

B : "Ça y est j'ai compris!" Il est un peu mystérieux, je le comprends pas bien moimême /.../

Mo : /.../ un peu de FRAYEUR mais en même temps avec une impression de SOULAGEMENT, à la fin.

Ma : Ça ne me paraît pas naturel non plus. C'est comme si c'était joué.

$\mathrm{P}$ : Surprise. /.../ on vient de voir un truc qu'on s'attendait pas. Plutôt une bonne surprise.

21 (4c) ah [?a.]

signifiant du signe vocal : occlusive glottale initiale, voyelle semi-longue, Fo modulé montant-descendant $\mathrm{MB}>\mathrm{M}>\mathrm{MB}$ (la descente est très brève)

interprétations données :

CRH : "Là, ça M'EMMERDE. Si j'avais su..."

Ma : Y a un peu de DÉCEPTION; ça tombe un peu à plat quoi; il aurait bien voulu que ce soit autrement et puis malheureusement, ça n'a pas été comme il souhaitait.

$\mathrm{P}:$ /.../ mauvaise surprise.

(4d) ah [?a]

signifiant du signe vocal : occlusive glottale initiale, voyelle brève avec rapprochement forcé des commissures (léger) - ce qui correspond à une partie du geste articulatoire de la labialité de type vocalique - ; Fo plat B

interprétations données :

CRH : "Y a plus rien à dire, même si cela ne ME PLAîT PAS". Il comprend que ce qu'il vient d'entendre est définitif et ne supporte aucun commentaire, ni remarque, ni a fortiori contradiction.

Ma $: \backslash \ldots \backslash$ je vois pas si y a une intention derrière.

23 (4e) ah $\left[a^{\tilde{\varepsilon}}\right.$. $]$

signifiant du signe vocal: voyelle semi-longue partiellement nasalisée; Fo modulé montant $\mathrm{MH}>\mathrm{H}, \mathrm{dB}^{-}$

interprétations données :

$\mathrm{CRH}$ : Il pose une question sous l'angle d'une demande de confirmation.

$\mathrm{B}$ : Interrogatif, avec une pointe de doute. /.../

Ma : Là il questionne. Il n'a pas compris. Il exprime qu'il faudrait peut-être qu'on lui précise des choses.

Mo : /.../ interrogatif /.../ 
(5) tableau récapitulatif des traits pour les "ah" du corpus

\begin{tabular}{|l|l|l|l|l|l|l|l|}
\hline quantité & labialité \\
vocalique & vocalique & $\begin{array}{l}\text { timbre et } \\
\text { tension } \\
\text { articulatoire }\end{array}$ & voisement & $\begin{array}{l}\text { consonne } \\
\text { non } \\
\text { phonol. }\end{array}$ & $\begin{array}{l}\text { hauteurs } \\
\text { tonales = Fo } \\
\text { (par rapport à la } \\
\text { tessiture du } \\
\text { locuteur) }\end{array}$ & $\begin{array}{l}\text { (par } \\
\text { rapport } \\
\text { à la base } \\
\text { du } \\
\text { locuteur }\end{array}$ \\
\hline$(4 a)$
\end{tabular}

Pour l'interprétation du sens de ces énonciations d'une seule syllabe elle-même constituée uniquement d'une voyelle (cf. Rittaud-Hutinet C. 2017 /sous presse/), le seul guide est le signifiant du signe vocal et donc les particularités acoustiques entendues (à lire dans les tableaux (3) (5)).

\subsubsection{Analyse qualitative des 'traductions' : concordance, ou divergence?}

S'agissant des contenus, que nous apprennent ces 10 exemples? Rappelons que nous sommes en présence d'énonciations hors situation, ce qui est d'ailleurs souligné ici et là par les personnes interrogées.

27 Ce qu'on observe d'abord c'est que chacune d'elles replaçant, involontairement bien sûr, dans un cotexte et un contexte qui lui sont propres la version effectivement entendue, les réponses montrent :

- parfois un accord complet sur le sens pragmatique dégagé, avec identité lexicale (au minimum un mot), comme : en (4b) CRH, Ma et Mo ; en (2c) CRH et B ; en (4e) CRH, B, Ma et Mo ; en (2e) Mo et CRH ;

- mais le plus souvent une grande convergence: 
* soit, d'une part comme en (2a) - avec une forte proximité entre CRH et Mo - par une description explicite sur la recréation mentale de contexte, et de l'autre au travers de paroles rapportées, comme : CRH en (2b), (2c), (4a), (4c), (4d); B et CRH en (4b); Mo et CRH en (2e) ;

* soit avec des termes expliquant le ressenti du destinataire (références sensorielles ou affectives) - même avec des mots différents -, comme : en (2a) CRH, B et Mo ; en (2e) $\mathrm{CRH}$, B et P ; en (4a) CRH et Mo ; en (4b) CRH et Mo ; en (4c) CRH et Ma, sachant que les mots choisis peuvent relever de niveaux de langue différents, comme en (4c) CRH et P ; * soit encore, comme en (2c) chez Ma, avec quelques mots sur l'objectif du parleur.

En outre B renvoie à une ou deux occasions à sa position "des deux côtés de la barrière" - comme diseur et comme interpréteur -, ainsi en (2a).

Mais il arrive aussi qu'on ne sache pas si le testé parle du signifié implicite tel qu'il l'a compris ou de son avis sur la prestation du diseur (B), notamment : en (2c), B, Ma et P.

Parfois encore le testé se trouve dans l'embarras. Dans ce cas,

- soit, tel Ma en (4a), il donne successivement 2 interprétations divergentes,

- soit, comme Ma en (4d), il s'avoue perdu.

Evidemment, la difficulté du travail de mise en mots, quand il faut passer à l'explicite, donc le manque de sûreté du dit consécutif à la complexité de la verbalisation est visible ici et là. On relève :

- pour un item, jusqu'à 6 ré-écoutes demandées, parfois même alors que la réponse de premier jet était parfaitement adéquate ;

- des tournures comme : "comment dire" ;

- des modalisations (importantes chez certains, moins pour d'autres) avec des formes telles que : "peut-être, plutôt, pourrait, un peu, pour moi, dirais, paraît, moi j'entends ça comme, un peu, quelque chose de, presque, je trouve, un certain, l'impression de/ que" ;

- des changements d'avis.

Enfin, il faut souligner que les testés savent faire des remarques sur la forme des items :

- par des comparaisons, ainsi B, Mo, P et CRH en (2b) et ( $4 \mathrm{~b})$,

- ou par une allusion/un renvoi au sous-entendu, avec des tournures comme: "làderrière, là-dedans, intention derrière".

Pour conclure à propos de ces exemples, si les signifiés de ces lexies-phrases sont à l'évidence le fait de la prosodie et seulement de la prosodie, en revanche il est difficile ici ou là de dire si on a affaire à un seul signe vocal ou à une combinaison de plusieurs signes vocaux.

\section{2. "vous c'est vous"}

Passons à 6 occurrences de la réplique : "vous c'est vous", entendues dans 2 films. Là aussi la diversité des dictions est grande, manifestant que les acteurs tentent de se rapprocher de situations où de 'vrais' locuteurs se trouvent en interaction langagière dans des échanges non guidés. Et ces variations ont bien sûr pour but de modifier les effets de sens.

a) Pour le premier extrait - tiré de: Entrée des artistes, de Marc Allégret, $1938^{7}$ (cf. extrait audio 6 joint) -, l'action se situe dans un cours de théâtre; un étudiant et une étudiante sont en train de jouer une scène devant la classe. Mais le professeur n'est pas 
satisfait de l'interprétation du jeune homme, et lui demande à 3 reprises de recommencer pour donner plus de réalisme à sa diction.

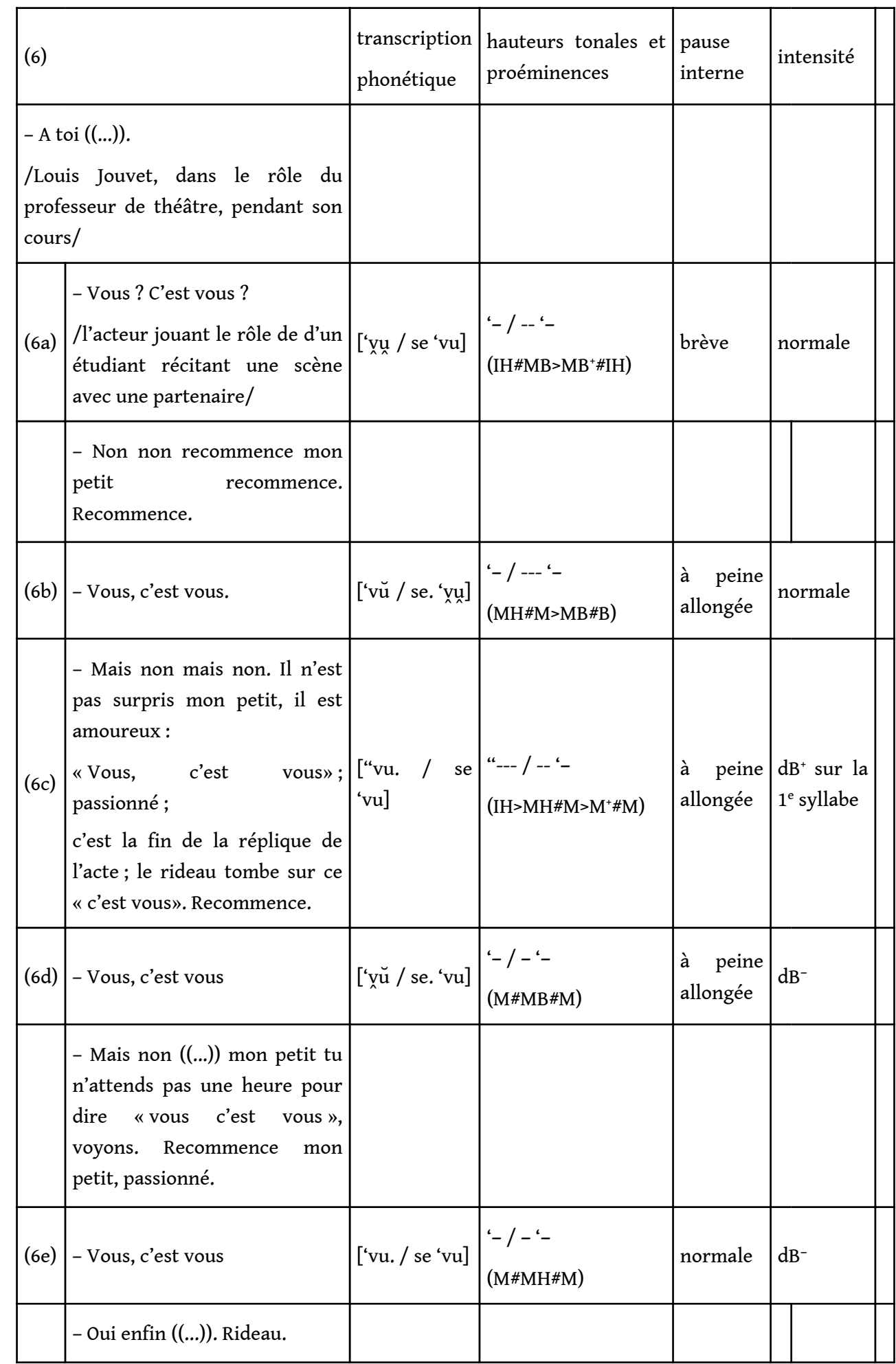

- La première version de l'étudiant, en (6a), manifeste une surprise, quelque chose qu'on pourrait traduire par exemple par : "Ah, tiens, je ne m'attendais pas à vous voir ici !" 

- (rire) C'est pas une alliance c'est une bague. Elle est trop large elle tourne toujours.

/Jean Marais, dans le rôle d'Alain Ginestier/

- Vous ?! ? C'est vous ?!

/Simone Renant, dans le rôle de Marie Ange/

- Comment, je comprends pas.

- Votre nom, dites-moi votre nom.

- Mais... Ginestier! Alain Ginestier pourquoi?

Nous sommes en présence de la réalisation d'un signe vocal très classique et courant à l'oral spontané. Abrégé en $<\mathrm{In}>^{9}$, son signifiant se développe sur tout le groupe prosodique :

- sur l'ensemble de l'énoncé : $d B$ augmentée par rapport au niveau moyen du locuteur - sur les 3 dernières syllabes : ${ }^{*}$ hauteurs tonales - pénultième, Fo plat IB - syllabe finale, Fo plat $\mathrm{H}$ à SH

* quantité : - dernière syllabe, toujours [V], quelle que soit la constitution syllabique.

Avec cette façon de prononcer "vous c'est vous", la femme exprime à la fois une surprise très grande et un scepticisme non moins important. Elle n'arrive pas à croire ce qu'elle vient de voir, à savoir la bague qui est au doigt de son interlocuteur : 
assurément, pour elle, ce n'est pas lui qui était censé porter ce bijou. D'où la question posée, laquelle est concomitante à la combinaison d'un étonnement extrême avec une incrédulité radicale.

\subsection{Dans une conversation non préparée} où les interlocuteurs se trouvent dans une situation très différente des exemples précédents: ce passage permettra d'examiner ce que font les interactants pour introduire et décliner un méta-discours, sachant que pour cela, selon l'intention exacte de l'énonciateur, au moins 2 signes vocaux différents peuvent être convoqués.

a) Le signe vocal abrégé en $<\mathrm{Su}>^{10}$, comme dans l'exemple (8), marque un accessoire du développement qui ne peut pas souffrir de délai pour être dit; sa transposition lexicale serait approximativement: "il s'agit d'informations indispensables, qui rendront plus clair et compréhensible ce que je vais dire ensuite" ${ }^{11}$.

Donc, sous forme d'inclusion - grammaticale ou non-grammaticale -, et dans le désir d'obtenir l'acquiescement du destinataire, $<\mathrm{Su}>$ permet de situer les circonstances, d'expliquer les causes proches ou lointaines, de donner des arguments anticipés quand on prévoit les contradictions possibles -, de prouver la légitimité de ce qu'on va dire, ou même de profiter de l'occasion pour parler de quelque chose qui n'a que peu de rapport avec l'objet actuel de discours, mais qu'on craint d'oublier par la suite, en raison notamment des dérives thématiques des interlocutions 'libres'.

(8)1'33"12 B- (il) y a eu l(e) salon du prêt à porter à Pa'ris 'là / c(e) week-'en(d) / et paraît $t$-il / parce que j’ai des gens bien placés qui y sont a'llés paraît $t$-il

\section{$:<\mathrm{Su}>$}

que :: / la 'mode c'est:: / c'est:: / c'est 'tout / la cou'leu :r des couleurs qui n(e) s'assemblent 'pas : / \...।

En (8), si B dit, par avance, de qui il tient l'opinion qu'il va développer, c'est parce qu'il trouve indispensable de prouver la validité de ce qu'il va rapporter. Dans cet exemple, le sens est transmis à la fois par des marques lexicales : "parce que", et par les marques vocales de $<$ Su $>$.

L'inclusion peut contenir un ou plusieurs groupes prosodiques, sur le(s)quel(s)

s'applique le signifiant vocal de $<\mathrm{Su}>$, constitué des traits suivants :

- une pause initiale (souvent),

- une accélération du débit (sauf sur la dernière syllabe de chaque groupe),

- un schéma intonatif incident

- parfois une pause subséquente.

b) L'exemple (9) offre une situation encore plus intéressante, car un $<\mathrm{Su}>$ y est suivi d'un autre signe vocal, abrégé en $<$ Sol $>^{13}$. Le but de l'emploi de $<$ Sol $>$ est lui aussi de signaler une inclusion méta-discursive, mais avec un objectif partiellement différent de celui de $\langle$ Su>. En effet, avec <Sol>, l'énonciateur éprouve le besoin de faire un commentaire pour clarifier le sens à donner $\mathrm{au}(\mathrm{x}) \operatorname{mot}(\mathrm{s})$ ou à la/aux phrase(s) qui précède(nt), mais il ne le fait pas dans un désir de consensus avec l'interlocuteur; au contraire, c'est uniquement parce qu'il réfléchit tout en parlant à la signification erronée que l'interlocuteur va peut-être donner à ce qu'il vient de prononcer. Avec cette sorte de redéfinition, son but est seulement d'être compris au plus juste. Bien qu'il

Corela, HS-20 | 2016 
soit en partie différent de l'aparté théâtral, ce phénomène lui est néanmoins assez comparable.

51 (9) $\mathrm{E}^{-14} \backslash \ldots \backslash$ oh ben : 'lui il est mal élevé comme 'tout / son profe'sseu :r aujour'd'hui

$\mathrm{i}(\mathrm{l}) \mathrm{lu}(\mathrm{i})$ a 'dit que : : / enfin son professeur leur donne les l(e)'çons : / la l(e)çon

début de $<$ Su> :

une fois qu'ils "l'ont : 'faite / qu'il leur donne les : : les impri'més : / alors il lui

: fin de $<\mathrm{Su}>$

$:<$ Sol>

a 'dit que c'était "pas : 'bien : qu'i(l) fallait qu'il leur 'donne a'vant : \...।

52 En (9),

- <Su> permet à $\mathrm{E}$ de décrire le "quand" et le "quoi" :

* dans un premier temps, à la lecture, le sens du passage marqué <Su> semble clair, grâce à "enfin", qui fait attendre une reformulation correctrice ;

mais :

* "professeur" est un sujet grammatical, alors que juste avant il était agent : "i(l) $\underline{\text { lu(i) a }}$ dit" - même s'il faut évidemment tenir compte de l'erreur grammaticale de la toute première formulation : "son professeur" au lieu de : "à son professeur".

A partir de "enfin", il s'agit donc en réalité d'une explication a priori qui est censée permettre au destinataire de comprendre ce qui sera dit après.

- <Sol> suit directement <Su>. Là non plus le passage concerné, "qu'il leur donne les les imprimés", ne pourrait pas être correctement compris avec la seule composante lexicale :

* certes, cela commence par une duplication d'une partie de ce qui précède : "qu'il leur donne" redit "son professeur leur donne" - avec, là aussi, une erreur grammaticale ; on suppose que "qu'il leur donne" est précédé dans l'esprit de l'émetteur par "c'est une fois qu'ils l'ont faite" ;

* mais la suite, "les les imprimés" veut faire comprendre qu'il ne s'agissait pas pour l'enseignant de "donner la leçon", mais de donner "les imprimés à propos de la leçon".

54 Le signifiant du signe vocal <Sol> se développe sur l'ensemble de l'énoncé marqué (qui, contrairement à $<\mathrm{Su}>$, ne contient jamais plus d'un seul groupe prosodique, mais dont le nombre de syllabes n'est pas limité). Il est réalisé avec :

- une pause initiale (souvent),

- un Fo très légèrement descendant et plus bas que celui du groupe précédent - en (9), la hauteur tonale s'entend comme un deuxième niveau de parenthèse -,

- une pause subséquente.

\section{Comment agir en FLE ?}

Les 3 types d'exemples analysés montrent assez clairement les conséquences de la composante vocale sur le message délivré, et donc l'importance de l'acquisition de signes vocaux par les apprenants en français langue étrangère (cf. Frota \& al. (éds) 2011, Gomez M. R. \& al. (éds) 2010, Rittaud-Hutinet C. 2013, Sanz C. \& al. 2011).

Mais certains enseignants peuvent s'interroger sur les manières de procéder, et entre autres se demander d'une part quelles habiletés ils ont à mettre en œuvre pour réaliser aisément le 'ton' juste, quand ils sont amenés à le produire/reproduire eux-mêmes et 
de l'autre, parmi les procédures, lesquelles sont les plus efficaces pour leur classe (selon les niveaux, les âges/sexes, les objectifs, le temps, etc.).

\subsection{Des savoir-faire de l'enseignant}

Il nous faut bien admettre qu'en tant que simples locuteurs de français langue première nous ne faisons que rarement attention aux particularités intonatives des signes vocaux - car la plupart du temps, ce qui est normal, nous nous attachons au signifié. Aussi, pour les enseignants qui désireraient contrôler qu'ils savent discerner clairement et interpréter avec justesse les signes vocaux qu'ils désirent faire pratiquer dans leur classe, je propose une vérification compétencielle, qui s'effectue avec deux tests et une pratique méta-langagière :

a) test 1 : après avoir écouté un énoncé enregistré (de préférence bref), nommer les hauteurs de la voix (Fo) pour chaque syllabe, en termes simples: "c'est haut, bas, moyen", "c'est plat, ça monte, ça descend", en exécutant le geste correspondant avec la main. Se fait évidemment sous contrôle, pour vérifier que le geste et les mots coïncident ;

b) test 2 : par groupes de deux, mise en situation et invention d'un dialogue très court, deux répliques dont la seconde est formée d'un seul et unique mot, par exemple: "Oui », le sens de celui-ci devant être complété ou modifié par la prosodie réalisée. Jouer ensuite la scène devant un 'public'; l'objectif est atteint si les réactions correspondent à ce que l'énonciateur 'voulait dire' ;

c) démarche paraphrastique : à partir d'un extrait d'enregistrement dont le sens implicite est porté par la couche vocale, reformuler verbalement, avec autant de phrases que nécessaire, le sens pragmatique dégagé. Le but est de s'habituer à avoir clairement présent à l'esprit les signifiés des signes vocaux (mais seulement à l'esprit, car on n'aura pas à les faire apprendre en tant que tels à la classe).

\subsection{Choisir les signes vocaux}

Dans ce genre d'activité, le plus difficile est de se limiter. Il serait en effet aussi dommage de multiplier les cas de figure que de ne rien faire. Pour les choix à faire, on retrouve des principes qui gouvernent l'enseignement des autres aspects de la langue :

- la spécificité et le consensus ; c'est-à-dire des signes vocaux qui possèdent un signifié pragmatique facile à décrire précisément et qui est accepté par l'ensemble de la communauté langagière de la langue-cible ;

- la lisibilité ; pour que les apprenants appréhendent facilement les signes vocaux, proposer ceux dont la forme acoustique et le signifié sont bien différenciés les uns des autres ;

- la quantité et le degré de fonctionnalité ; travailler peu de formes à la fois, en commençant d'abord par celles qui sont très couramment usitées dans le langage quotidien.

\section{3. À propos des techniques}

Ce type d'apprentissage sollicite un investissement important de la part des apprenants. Aussi est-il important de les prémunir contre les idées reçues dont certains ouvrages - toujours utilisés actuellement - continuent de se faire l'écho, et tout 
particulièrement la croyance que "les erreurs de perception révèlent souvent des défauts au niveau de l'émission" (Pagniez-Delbart T., $1^{\mathrm{e}}$ éd. 1995, $2^{\mathrm{e}}$ éd. 2000), alors que la réalité est inverse: dans la reproduction, les fautes sont liées à une mauvaise perception.

L'exploitation dans la classe de cette dimension de la langue a également pour but de modifier les attitudes des apprenants face à leur apprentissage, en leur offrant l'opportunité d'exprimer ensuite facilement leurs propres opinions et ressentis. Logiquement donc, pour qu'ils y 'croient' en les disant, l'idée est, là comme pour les autres domaines de la langue, de faire accepter aux apprenants de 'jouer' les signes vocaux, en exploitant la plus grande variété possible de ressources ludiques et affectives : jeux de rôles, chansons, jeux de mots, théâtre, jeux de société, situations quotidiennes d'opposition, de connivence, de colère, de charme, de persuasion, de scepticisme, etc. (cf. G. \& al. 2014, Charalampos T. \& al. 2014, Jarmai E. M. 2010, RittaudHutinet 2003, 2009, 2011a, Sierra J. \& al. 2014). La contextualisation, encore plus importante ici qu'ailleurs, bénéficie souvent de l'adjonction de l'intégration corporelle à divers niveaux : mimiques, gestes, déplacements.

61 Evidemment, quand on n'a pas à sa disposition d'enregistrement de tel ou tel signe vocal - et donc qu'on doive être soi-même l'énonciateur des exemples. Là, c'est l'enseignant qui se met en scène, avec 2 difficultés : a) sachant qu'il y a des synonymes dans les signes vocaux comme dans le vocabulaire, la contrainte est de demeurer univoque, en conservant toujours la même diction - donc le même signifié pragmatique - pour chaque cas ; b) il n'est pas toujours évident de se mettre 'dans la peau du personnage', condition indispensable pour être crédible, mais qui n'est pas le cas rappelons-le dans l'exemple (6) supra: l'impression donnée par la diction est mauvaise, parce que le professeur de théâtre qui donne une indication à son élève ne réalise pas lui-même ce qu'il préconise.

\section{Eléments de conclusion}

La couche vocale se montre en français un constituant indispensable pour l'échange oral. En effet le parleur recourt beaucoup à la multimodalité et singulièrement aux unités linguistiques sonores que sont les signes vocaux, sans lesquels quantité de lieux de nos discours demeureraient ambigus ou amputés d'une partie importante du sens que nous voulons leur donner, de ce pour quoi nous les proférons.

Pourtant, dans l'enseignement du français comme langue étrangère, l'implicite de l'énonciation transmis par la prosodie signifiante ${ }^{15}$ est fréquemment négligé, alors que toute pratique d'enseignement est confrontée à la réalité de cette strate de la langue, les erreurs d'intonation freinant ou empêchant la compréhension.

Avec les exemples analysés, nous avons fait les principales observations suivantes :

- les signes vocaux démontre tout particulièrement leur utilité avec les énoncés très brefs, qu'ils soient réalisés ou non en contexte, car même alors leur efficacité est totale ;

- ils exploitent un large éventail d'indices acoustiques, et ce dans une variété de combinaisons conséquente,

- et les effets de sens sont eux aussi très diversifiés ; pour les traduire verbalement, les termes relevés pour les exemples analysés ici vont entre autres de plaisir à angoisse en 
passant par incrédulité, d'inquiétude à humour, de bonne ou de mauvaise surprise à admiration ou à demande de confirmation, d'énervement à soulagement ou à déception.

On constate donc que les signes vocaux constituent une sorte de dictionnaire sonore pour tout ce qu'on veut sous-entendre. Des ressources qui apparaissent indispensables aux apprenants en français comme langue étrangère dès lors qu'il s'agit pour eux d'affiner leurs stratégies interlocutives et en même temps d'être plus adéquats par rapport aux contraintes sociétales et relationnelles, et donc pour que leurs communications langagières avec les Français s'établissent et se maintiennent à un degré satisfaisant d'acceptabilité, de recevabilité, c'est-à-dire de bonne interprétation et d'intercompréhension plus aisée.

D'où, pour préparer les apprenants à les utiliser de façon efficace, l'importance pour les enseignants de programmer des activités adaptées à cette facette de l'interlocution.

\section{conventions de transcription (limitées à ce qui apparaît dans les exemples)}

$67[?]=$ consonne occlusive glottale

$[\mathrm{h}]=$ consonne fricative glottale

[o] $=$ o ouvert

$[\breve{V}]=$ voyelle très brève

[u $]$ = voyelle/consonne partiellement voisée

[.] = semi-long

$[::]=$ très long

[]$=$ ultra-long

[VṼ] = nasalisation partielle avec degré d'aperture intermédiaire

$><$ = rapprochement forcé des commissures ( = labialité contrariée de type vocalique)

<> = écartement forcé des commissures ( = labialité forcée de type vocalique)

$\mathrm{dB}^{+}=$augmentation d'intensité

$\mathrm{dB}^{-}=$diminution d'intensité

$\mathrm{dB}^{++}=$augmentation forte d'intensité

$\left[{ }^{\prime}\right]$ = accent tonique (groupe prosodique)

["] = accent d'intensité

$<\mathrm{X}>=$ signe vocal

$-->>$ début de la zone d'application de signe vocal

------ = zone d'application de signe vocal

>---- = fin de la zone d'application de signe vocal

$((. .)$.$) = incompréhensible$

$\mathrm{X}>\mathrm{Y}=$ ton modulé

[\#] = limite syllabique

échelle des valeurs tonales ( = gamme dans la tessiture du locuteur):

$\mathrm{SH}=$ supra-haut ( = hors de la gamme habituelle du locuteur)

$\mathrm{H}^{+}=$très haut

$\mathrm{H}=$ haut

$\mathrm{IH}=$ infra-haut

$\mathrm{MH}=$ moyen haut

$\mathrm{M}=$ moyen

$\mathrm{MB}=$ moyen-bas 
$\mathrm{B}=\mathrm{bas}$

IB = infra-bas ( = hors de la gamme habituelle du locuteur)

\section{BIBLIOGRAPHIE}

Avanzi Mathieu \& Delais-Roussarie Elisabeth (2011), "Regards croisés sur la prosodie du français - des données à la modélisation", Journal of French Language Studies, Cambridge University Press, 2011, 21/1, 1-12

Barth-Weingarten Dagmar, Dehé Nicole \& Wichmann Anne (éds.) (2010a), When Prosody Meets Pragmatics, Emeralg Group Publishing Ltd, Studies in Pragmatics 8

Barth-Weingarten Dagmar, Reber Elisabeth \& Selting Margret (éds.) (2010b), Prosody in Interaction, John Benjamins, Studies in Discourse and Grammar Series 23

Beyssade Claire et al. (2004), "Prosody and Information in French", Handbook of French Semantics, F. Corblin \& H. de Swaert (éds.) CSLI

Brichet Cécile \& Aubergé Véronique (2003), "La focalisation en français : morphologie de la prosodie", in Aubergé V., Lacheret-Dujour A. \& Lœvenbruck (éds.), Actes des Journées prosodie 2001, C.N.R.S.-GDR 34 Phonologie/Institut de la communication parlée/CREST Expressive Speech Project, 67-74

Briet Geneviève, Collige Valérie \& Rassart Emmanuelle (2014), La prononciation en classe, P. U. Grenoble, coll. Les outils malins du FLE

Camp Elisabeth (2006), "Contextualism, metaphor and what is said", Mind and Language 21-3, 280-309

Carston Robyn (2009), "The explicit/implicit distinction in pragmatics and the limits of explicit communication", International review of pragmatics, 1, 35-62

Charalampos Tsatsarelis, Kress Gunther, Jewitt Carey \& Ogborn Jon (éds) (2014), Multimodal Teaching and Learning. The Rhetorics of the Science Classroom, Bloomsbury Publishing (formerly The Continuum International Publishing Group)

Cosnier Jacques \& Kerbrat Catherine (éds) (1987), Décrire la conversation, P.U. Lyon

Frota Sonia, Elordieta Gorka \& Prieto Pilar (éds.) (2011), Prosodic Categories : Production, Perception and Comprehension, Springer, Studies in Natural Language and Linguistic Theroy Series

Gabrys-Barker Danuta \& Bielska Joanna (éds) (2013), The Affective Dimension in Second Language Acquisition, Multilingual Matters, Second Language Acquisition Series

Godement-Berline R. (2014), L'emploi de la focalisation prosodique dans le jeu d'acteur", Nouveaux cahiers de linguistique française, 31, 129-139

Gómez Morón Reyes, Padilla Cruz Manuel, Fernández Amaya Lucía \& de la O Hernández López María (éds.) (2010), Pragmatics Applied to Language Teaching and Learning, Cambridge Scholars Publishing 
Hirschberg Julia (2002), "Communication and prosody : functional aspects of prosody", Speech Communication 36, 31-43

Jarmai Elizabeth Marie (2010), "L’aspect affectif dans la didactique du français langue étrangère", in Abecassis Michaël \& Ledegen Gudrun (éds), Les voix des Français. A travers l'histoire, l'école et la presse, Bern, Peter Lang, Modern French Identities Series 93, volume I, 187-200

Kissine Mikhail V. (2013), From Utterances to Speech Acts, Cambridge University Press

Lacheret-Dujour Anne et Beaugendre Frédéric (1999), La prosodie du français, éd. C.N.R.S.

Marti Luisa (2006), "Unarticulated constituents revisited", Linguistics and Philosophy 29, 135-166

Morel Mary-Annick \&. Danon-Boileau Laurent (1998), Grammaire de l'intonation. L'exemple du français, éd. Ophrys

Pagniez-Delbart T., À l'écoute des sons, Clé international, 2000 [1e éd. 1995] ; 2 vol. : les consonnes, les voyelles

Plantin Christian, Doury Marianne \& Traverso Véronique (éds) (2000), Les émotions dans les interactions, Lyon, Presses Universitaires de Lyon

Portes Cristel \& Bertrand Roxane (2011), "Permanence et variation des unités prosodiques dans le discours et l'interaction", Journal of French Language Studies, Cambridge University Press, 21/1, 97-110

Rittaud-Hutinet Chantal (1980), Le français, langue plurielle, P.U. Lille, 3 tomes

Rittaud-Hutinet Chantal (2003), "La pragmatique au service de la glottodidactique", in Kacprzak A. (éd.), Points communs : linguistique, glottodidactique, traductologie, Lodz, Wydawnictwo Biblioteka, 215-229

Rittaud-Hutinet Chantal (2006), "Les signes vocaux : au carrefour des strates d'interprétation des messages oraux", in Cyril Auran, Roxane Bertrand, Catherine Chanet, Annie Colas, Albert Di Cristo, Cristel Portes, Alain Régnier \& Monique Vion (éds.) Interface discours - prosodie, IDP 05, sur CDRom et sur le site : http://www.lpl.univ-aix.fr/ prodige/idp05/idp05_fr.htm

Rittaud-Hutinet Chantal (2008), "Consensus, conflits et négociations implicites", communication au Colloque international Les voix du français : usages et représentations, Oxford 3-5 septembre

Rittaud-Hutinet Chantal (2009a), "Le langage non verbal, ou : signes vocaux et sens du message", Revue de sémantique et pragmatique 19/20, 71-88

Rittaud-Hutinet Chantal (2009b), "Approche ludique de la variation", in Bertrand Olivier \& Schaffner Isabelle (éds.), Quel français enseigner ?La question de la norme dans l'enseignement/ apprentissage, Paris, éd. de l'École Polytechnique, 185-199

Rittaud-Hutinet Chantal (2011a), "Jouer à changer de voix : pistes pour une formation à l'alternance prosodique", in Clerc Stéphanie (éd.), (Mé)tisser les langues à l'école? Quels outils, quels curricula et quelles formations pour le développement du plurilinguisme à l'école ?, Cahiers de Linguistique, Revue de sociolinguistique et de sociologie de la langue française, 37/2, 151-162

Rittaud-Hutinet Chantal (2011b), "Prosodie et variation du sens", in Bertrand Olivier \& Schaffner Isabelle, Variétés, variations \& formes du français, éd. de l'Ecole Polytechnique, 93-109

Rittaud-Hutinet Chantal (2011c), "Oral spontané et prosodie en FLE", communication au Colloque international (Des)organisation de l'oral ? De la segmentation à l'interprétation, Rennes, 24-25 mars; à paraître 2015, Presses universitaires de Rennes, coll. Rivages linguistiques 
Rittaud-Hutinet Chantal (2012), "Cachettes du sens : l'oral à découvert", in Marillaud Pierre \& Gauthier Robert (éds), Ambiguités, CALS/CPST, Presses universitaires de Toulouse II-Le Mirail, 59-70

Rittaud-Hutinet Chantal (2013), "Focaliser sans mots en français langue étrangère : techniques pour apprenants et enseignants", communication au Colloque international : Langue française mise en relief(s) : aspects linguistiques, didactiques et institutionnels (AFLS 2013), Perpignan, 6-8 juin ; Actes à paraître 2017

Rittaud-Hutinet Chantal (2017), "Comment comprendre les non-dits ?", in Castagne Eric (éd.), Intercompréhension et analogie, (sous presse)

Rossi Mario, Di Cristo Albert, Hirst Daniel, Martin Philippe, Nishinuma Yukihiro (1981) : L'intonation. De l'acoustique à la sémantique, Klincksieck, études linguistiques XXV

Sanz Cristina \& Leow Ronald P. (2011), Implicit \& Explicit Language Learning, Georgetown univ. press

Sierra Juan Manuel, Lasagabaster David \& Doiz Aintzane (éds) (2014), Motivation and Foreign Language Learning. From theory to practice, John Benjamins, Language Learning \& Language Teaching Series 40

Stewart Dominic (2009), Semantic Prosody. A Critical Evaluation, Routledge (Taylor \& Francis), coll. Routledge Advances in Corpus Linguistics

Wennerstrom Ann (2001), The Music of Everyday Speech : Prosody and Discourse Analysis, Oxford Univ. Press

\section{NOTES}

1. Je préfère "prosodie signifiante" à "semantic prosody", car cela évite toute confusion avec la paire : sens sémantique $\sim$ sens pragmatique.

2. Simenon Georges [1932] (2003), L'Affaire Saint-Fiacre, Omnibus, collection "Tout Simenon" 17, p. 306. Le texte original ne présente pas d'italiques.

3. Fernand Rivers, 1937 ; avec notamment Raimu et Françoise Rosay.

4. Il m'a paru inutile de signaler ici l'accent tonique, (toujours sur la dernière syllabe du groupe prosodique), étant donné que l'on n'a qu'une seule syllabe. Seul l'accent d'intensité est signalé le cas échéant, car lui en revanche n'est pas automatique.

5. c'est-à-dire que cette partie du geste articulatoire du trait: + labial, de la labialité de type vocalique est contrariée. Naturellement, s'entend moins sur les unités contenant le trait : - labial. 6. c'est-à-dire que cette partie du geste articulatoire du trait: + labial, de la labialité de type vocalique est forcée. Naturellement, s'entend moins sur les unités contenant le trait : + labial.

7. Avec notamment : Louis Jouvet, Claude Dauphin, Janine Darcey, Odette Joyeux, Sylvie, Bernard Blier, Julien Carette, Noël Roquevert. L'extrait commence à 23'31".

8. Avec notamment : Simone Renant et Jean Marais. L'extrait commence à 52'.

9. $<$ In> comme : Incrédule.

Ce signe vocal et son abréviation, de même que les suivants, fait partie de ceux que nous avons déjà élicités au cours de nos recherches phonopragmatiques.

10. $<\mathrm{Su}>$ comme: Supplément d'information indispensable à la bonne compréhension de l'information centrale.

11. Morel M.-A. \& Danon-Boileau L. 1998 nomment cette séquence discursive le "cadre", au sein du "préambule" d'un "paragraphe oral", qui pour eux "délimite une zone de prédication" et doit 
être marqué pour être reconnu quand il se trouve après le support lexical disjoint au lieu d'être avant.

12. Les exemples minutés sont extraits du corpus in Cosnier J. \& Kerbrat C. (éds) 1987

13. $<$ Sol> comme : Soliloque.

14. corpus in Rittaud-Hutinet C. 1980

15. cf. note 1 .

\section{RÉSUMÉS}

C'est vers les enseignants de français comme langue étrangère qu'est orientée cette analyse : leur offrir encore plus de moyens pour qu'ils incluent la prosodie signifiante ${ }^{1}$ dans leur pédagogie, l'objectif étant l'acquisition et la maîtrise par les apprenants au moins des signes vocaux les plus courants, qui permettent de transmettre des sens différents avec les mêmes paroles, savoir-faire nécessaire en français pour comprendre et être compris des Français.

Dans ce but sont décrits dans cet article: a) quelques uns des sens pragmatiques apparaissant grâce à ces unités prosodiques que sont les signes vocaux, avec des exemples d'énoncés constitués pour certains d'énoncés plus ou moins longs et pour d'autres d'une simple interjection; b) quelques pistes vis-à-vis des savoir-faire et des techniques à appliquer dans la classe.

The aim is to propose to teachers of French as a Foreign Language some more ways to help their students to learn how to put prosodic features into practice. The objective is to enable students to learn an master at least a few of the vocal signs with which they can express different meanings, using the same words. In French, this skill is part of a savoir-faire that students must possess if they want to understand, and be understood by French speakers.

In this purpose the paper : a) describes in detail some of the prosodic units and the pragmatic meanings that appear with them, with examples in sentences or only an interjection; b) some (new) ideas for the what and how to teach vocal signs in the classroom.

\section{INDEX}

Keywords : spontaneous oral french, multimodality, semantic prosody, speech acts, interjections, teaching french speaking

Mots-clés : français oral spontané, multimodalité, prosodie signifiante, actes de langage, interjections, techniques pour la classe de français langue étrangère

\section{AUTEUR}

\section{CHANTAL RITTAUD-HUTINET}

chrit@wanadoo.fr

Université Paris 3-Sorbonne nouvelle, EA 7245 CLESTHIA 